\title{
NOUVELle
}

\section{Immunité humorale anti-adénovirus}

\section{Impact sur l'activation des cellules dendritiques}

Franck J.D. Mennechet, Karsten Eichholz, Thi Thu Phuong Tran, Eric J. Kremer

Institut de génétique moléculaire de Montpellier (IGMM), CNRS, université de Montpellier, Montpellier, France.

franck.mennechet@igmm.cnrs.fr
> Les adénovirus ( $A d$ ) sont des virus non-enveloppés à ADN double brin, responsables d'infections respiratoires, oculaires et gastro-intestinales. Si la plupart des infections sont résolues rapidement sans attention particulière, les adénovirus peuvent parfois conduire à des complications sévères chez des individus immunodéprimés. Au cours de la vie, nous rencontrons fréquemment de nombreux adénovirus (plus de 70 types infectant l'homme sont répertoriés) qui se transmettent généralement par gouttelettes aéroportées ou par simple contact avec des surfaces contaminées. L'infection conduit au développement d'une réponse immunitaire robuste qui nous protège efficacement. Cependant, si de nombreuses études décrivent la réponse immunitaire primaire dirigée contre les adénovirus, peu d'informations sont disponibles concernant l'interface entre la réponse immunitaire humorale (c'est-à-dire les anticorps) anti-Ad et les cellules présentatrices d'antigènes (CPA) lors de situations de réinfections ou d'infections persistantes. Le but de notre étude a été de mieux caractériser l'impact de la réponse adaptive humorale sur les CPA et de pouvoir, à terme, l'intégrer dans un contexte plus général de modulation de l'immunité antivirale.

\section{La face cachée des anticorps anti-} adénovirus

La présence d'anticorps anti-Ad conduit à la formation de complexes immuns ( $\mathrm{Cl}$ ) qui permettent d'empêcher l'attache- ment du virus à ses récepteurs, le désassemblage de la capside, ou faciliter son élimination par phagocytose.

Les $\mathrm{Cl}$ peuvent également être pris en charge par les CPA via les récepteurs pour la région $\mathrm{Fc}$ des immunoglobulines $(F c \gamma R)$ que ces cellules expriment. Par conséquent, la présence d'anticorps anti-Ad peut considérablement modifier l'efficacité thérapeutique d'un vecteur dérivé d'Ad. Ces vecteurs, fréquemment utilisés en thérapie génique et en vaccination, ont l'avantage de ne pas s'intégrer au génome, tout en permettant une expression stable du transgène dans certains tissus [1]

$(\rightarrow)$. Notre étude

montre que des

complexes immuns

$\rightarrow$ Voir la Synthèse de A. Rossi et

A. Salvetti, $m / s n^{\circ} 2$, février 2016, page 167

formés entre adénovirus et anticorps anti-Ad $(\mathrm{Cl}-\mathrm{Ad})$ déclenchent une forte réponse immunitaire naturelle conduisant à la pyroptose des cellules dendritiques (CD) [2]. La pyroptose [3], ou «mort cellulaire dépendante de la caspase $l »$, est une forme de mort cellulaire programmée jouant un rôle crucial dans l'inflammation et dans l'élimination de certains pathogènes intracellulaires (Figure I). L'impact, chez l'hôte, de la pyroptose aboutissant in fine à la mort des CD reste encore inconnu, mais ce phénomène pourrait, en partie, expliquer les complications parfois observées lors de l'utilisation de vecteurs Ad. Dans notre étude, nous proposons un mécanisme permettant d'expliquer comment les anticorps modifient la réponse immunitaire contre les adénovirus chez les individus immunocompétents ou déficients, lors de situations de réinfection par le virus, d'utilisation de vaccins dérivés des adénovirus, ou au cours de traitements thérapeutiques utilisant des immunoglobulines.

\section{Caractéristiques des complexes immuns-adénovirus}

Les $\mathrm{Cl}$ sont le résultat de l'association macromoléculaire entre un antigène et un anticorps. Ils peuvent, parfois, se distinguer en fonction des quantités d'antigène et d'anticorps qui les constituent. L'association d'adénovirus de type 5 ( $\operatorname{dd} 5$ ) avec un sérum humain contenant des anticorps neutralisants (NAc) conduit à la formation de complexes « $\mathrm{Cl}-\mathrm{Ad}$ », d'une taille voisine de $200 \mathrm{~nm}$ de diamètre, capables d'activer la maturation/activation des CD. L'analyse plus précise des $\mathrm{Cl}$-Ad montre que ce sont principalement les anticorps dirigés contre les hexons ${ }^{1}$ de la capside de l'adénovirus qui sont à l'origine de la formation et de la stabilisation de ces complexes. Ces $\mathrm{Cl}$-Ad sont d'ailleurs fréquemment retrouvés dans la circulation sanguine lors d'une virémie, ou encore, post-mortem, dans les tissus de patients, à la suite d'une virose mortelle. Contrairement à ce que l'on observe chez la souris, le vecteur adénovirus par luimême n'a que très peu d'effet direct sur l'activation des CD humaines.

${ }^{1}$ L'hexon est la protéine majoritaire de la capside, formant les 20 faces triangulaires du virus. 


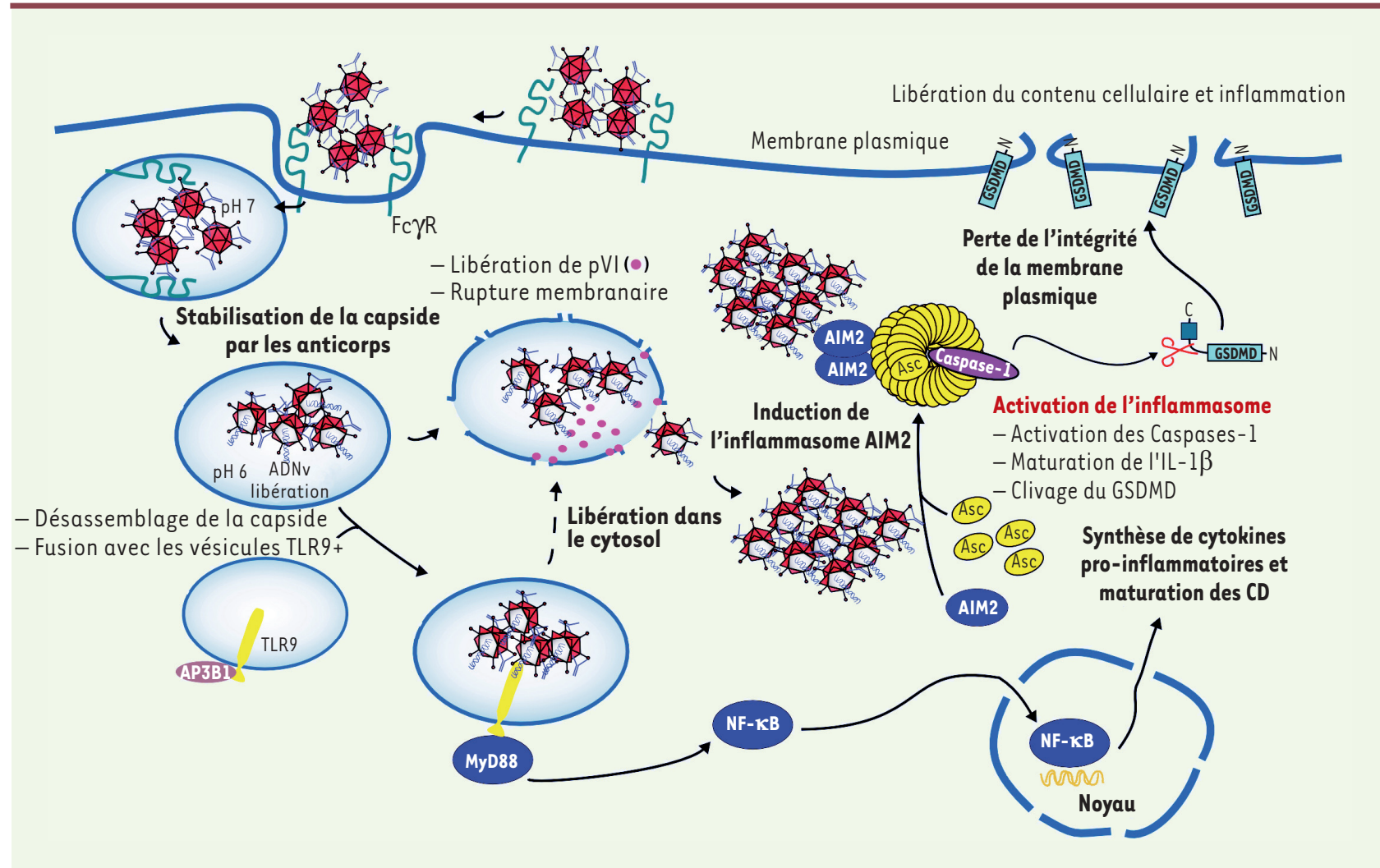

Figure 1. Prise en charge des complexes immuns - adénovirus (IC-Ad) par les cellules dendritiques : modélisation des évènements conduisant à la mort cellulaire par pyropotose. AP3B1 : adaptor-related protein complex 3 beta 1 ; MyD88 : myeloid differentiation primary response protein 88 ; AIM2 : absent in melanoma 2 ; ASC : apoptosis-associated speck-like protein containing a caspase recruitment domain; GSDMD : gasdermin-D; TLR9 : Toll-like receptor 9 ; CD : cellules dendritiques; PVI : protéine de capside interne VI.

Interaction complexes immunscellules dendritiques : que devient le virus?

Les CD ont pour rôle de capturer, d'apprêter et de présenter les antigènes aux lymphocytes $T$. Ce sont des acteurs capitaux de la réponse immunitaire qui font le lien entre l'immunité innée et l'immunité adaptative. Les CD vont déclencher, orienter, mais aussi en partie moduler la réponse lymphocytaire $\mathrm{T}$. Au cours de la prise en charge des $\mathrm{Cl}$ par les $C D$, via les $F c \gamma R$, les récepteurs de reconnaissance de motifs moléculaires (les PRR, pour pattern recognition receptor) de type Toll, tels que le TLR9 vésiculaire (Toll like receptor 9$)^{2}$, sont généralement mis à contribution [4]. Reposant sur des approches pharmacologiques et microscopiques, nos études

${ }^{2}$ Le TLR9 reconnaît des motifs CpG présents dans l'ADN. montrent qu'une fois captés par les $C D$, les $\mathrm{Cl}$-Ad vont interagir avec le TLR9 et déclencher ainsi une production massive de cytokines pro-inflammatoires. Ce processus nécessite un désassemblage partiel du virus afin de libérer une partie du génome pour l'exposer au TLR9. La capside sort ensuite de la vésicule pour rejoindre le compartiment cytoplasmique de la cellule hôte par un mécanisme dépendant de la protéine de capside interne VI ( $p V I$, protéine impliquée dans la destruction des membranes vésiculaires) [5]. Les $\mathrm{Cl}-\mathrm{Ad}$ initient ensuite le mécanisme de pyroptose dans le cytoplasme. Nos observations établissent un changement drastique de la taille et la granulosité des CD sous I'action des $\mathrm{Cl}$-Ad. Grâce à des adénovirus marqués, nous avons établi que ces modifications morphologiques sont directement liées à la perte de l'intégrité de la membrane plasmique des cellules. L'utilisation de capsides vides et d'un virus mutant pour la protéine pVI (n'exprimant plus cette dernière) démontre l'implication du génome viral et de $\mathrm{pVI}$ dans la régulation de l'homéostasie membranaire de la cellule hôte. Les premières études sur l'interaction des adénovirus (en l'absence d'anticorps) avec l'inflammasome ${ }^{3}$, chez l'homme, suggèrent l'implication du complexe NLRP3 (NOD-like receptor family, pyrin domain containing 3) [6]. Cependant, nos travaux montrent que, quand les adénovirus sont opsonisés par les anticorps, l'activation de l'inflammasome fait intervenir un mécanisme dépendant du complexe AIM2 (absent in melanoma 2) / ASC (apoptosis-associated

${ }^{3}$ L'inflammasome est un complexe protéique exprimé dans la lignée myéloïde et impliqué dans l'immunité innée. 
speck-like protein containing a caspase recruitment domain) [7] $(\rightarrow)$. AIM2 est un PRR qui détecte I'ADN double brin dans le cytoplasme et recrute la protéine adaptatrice $(\rightarrow)$ Voir la Synthèse de Y. Jamilloux et $T$. Henry, $m / s n^{\circ} 11$, novembre 2013, page 975
ASC. Nos approches moléculaires, cellulaires et pharmacologiques permettent de proposer un mécanisme expliquant la perte de l'intégrité membranaire des $C D$, la libération massive de cytokines inflammatoires, la maturation/activation des CD, puis leur mort par pyroptose (Figure 1). Ce modèle fait intervenir la stabilisation de la capside Ad par les anticorps neutralisants qui perturbent le devenir vésiculaire des $\mathrm{Cl}-\mathrm{Ad}$, conduisant à la détection du génome viral par le récepteur TLR9. L'échappement du $\mathrm{Cl}$-Ad de son compartiment vésiculaire conduit ensuite à l'induction de l'inflammasome par un mécanisme indépendant du complexe NLRP3, mais étroitement associé à AIM2/ASC. In fine, on observe la formation de pyroptosomes ${ }^{4}$ et la dégradation de l'intégrité de la membrane plasmique par le système GSDMD (gasdermin-D) [8].

\section{Conséquences sur la modulation de l'immunité et la thérapie}

Notre modèle propose l'intervention du complexe AIM2/ASC comme inducteur de l'inflammasome par les assemblages $\mathrm{Cl}-\mathrm{Ad}$. Les cellules dendritiques que nous avons utilisées, dérivent de monocytes sanguins humains provenant d'une multitude de donneurs. Les monocytes représentent $10 \%$ des leucocytes chez l'homme et sont systématiquement confrontés à des phénomènes de recrutement et d'activation lors du processus inflammatoire. Sous l'influence des pathogènes ou de l'inflammation, ces cellules peuvent se différencier en macrophages ou en cellules dendritiques (CD) et ainsi initier et moduler la réponse immunitaire adaptative. Les caractéris-

${ }^{4}$ Un pyroptosome est un assemblage supramoléculaire de dimères de protéines adaptatrices ASC. tiques uniques des CD dérivées de monocytes en font, selon nous, des candidats de choix pour analyser ex vivo la réponse immunitaire dirigée contre un pathogène. Il nous semble également pertinent de s'interroger sur l'intérêt physiologique d'un tel système. En quoi la pyroptose des CD par les IC-Ad profiterait-elle à I'hôte ou, inversement, au pathogène? La plupart des études suggèrent un effet pro-hôte qui permettrait de limiter l'invasion de l'organisme par le pathogène. La réponse immunitaire n'est cependant pas un système simple, linéaire et identique dans toutes les circonstances. Ainsi, certains pathogènes comme le VIH (virus de l'immunodéficience humaine) profitent de la pyroptose pour affaiblir la réponse immunitaire [9]. Détruire les CD par pyroptose limite aussi la capacité de développement de la réponse adaptative en contrôlant le nombre de cellules présentatrices (CPA). En revanche, la pyroptose est un événement fortement inflammatoire qui participe au recrutement et à l'activation des cellules immunitaires sur le site de l'infection. Dans le cas des adénovirus, l'infection est le plus souvent contrôlée, ce qui suggère un rôle plutôt protecteur de la pyroptose des CD par les Cl-Ad chez l'homme. Cependant, nous pouvons nous demander comment les adénovirus peuvent persister des années, voire des décennies chez l'hôte? L'environnement créé par la formation des $\mathrm{Cl}$-Ad et leurs actions sur les CPA n'auraient-ils pas d'autres fonctions, en particulier lors de situation d'infection persistante par des virus?

\section{Conclusion}

Nos travaux s'inscrivent dans un vaste cadre d'étude de l'immunité humaine dirigée contre les pathogènes. Mieux caractériser les interactions subtiles qui interviennent entre l'immunité humorale et les CPA pour chaque individu est capital. Cette étape est primordiale pour appréhender avec succès le traitement de complications virales apparaissant, par exemple, au cours des GVHd (ou réaction du greffon contre
I'hôte) ou lors de l'utilisation d'adénovirus pour la vaccination ou pour une thérapie génique. Le choix de vecteurs adénoviraux n'est en effet pas sans risque $[1,10]$. Par exemple, les essais de vaccination anti-VIH développés par le groupe Merck \& Co, Sharp \& Dohme $(M S D)^{5}$, utilisant le vecteur viral Ad5, ont brutalement été arrêtés en raison de son inefficacité, mais surtout d'une augmentation du taux de contamination par le VIH des patients séropositifs pour l'adénovirus de type 5 [11]. La présence d'anticorps anti-adénovirus s'est révélée directement liée à I 'échec du vaccin, mais aussi à l'augmentation des risques de contamination par le VIH [12]. Le virus Ad5 est un cas d'école. En effet, $90 \%$ de la population mondiale est séropositive pour cet adénovirus. Pour les autres types d'adénovirus, la séroprévalence reste souvent inconnue, mais est probablement très variable selon les régions du monde. $\diamond$

Anti-adenovirus humoral immunity: impact on dendritic cell activation

\section{LIENS D'INTÉRÊT}

Les auteurs déclarent n'avoir aucun lien d'intérêt concernant les données publiées dans cet article.

\section{RÉFÉRENCES}

1. Rossi A, Salvetti A. Intégration des vecteurs AAV et mutagenèse insertionnelle. Med Sci (Paris) 2016 ; 32 : 167-74.

2. Eichholz K, Bru T, Tran T, et al. Immune-complexed adenovirus induce AIM2-mediated pyroptosis in human dendritic cell. PLoS Pathog 2016 ; 12 : e1005871.

3. Miao \&, Leaf I, Treuting PM, et al. Caspase-1-induced pyroptosis is an innate immune effector mechanism against intracellular bacteria. Nat Immunol 2010 ; $11: 1136-42$.

4. Henault J, Martinez J, Riggs JM, et al. Noncanonical autophagy is required for type I interferon secretion in response to DNA-immune complexes. Immunity $2012 ; 37: 986-97$.

5. Wiethoff CM, Wodrich H, Gerace L, Nemerow GR. Adenovirus protein VI mediates membrane disruption following capsid disassembly. J Virol 2005 ; 79 : 1992 2000.

6. Barlan U, Griffin TM, McGuire KA, Wiethoff CM. Adenovirus membrane penetration activates the NLRP3 inflammasome. J Virol $2011 ; 85$ : 146-55.

7. Jamilloux Y, Henry T. Les inflammasomes : Platesformes de l'immunité innée. Med Sci (Paris) 2013 ; 29 : 975-84.

5 L'étude date de 2009 avant sa fusion (en 2009) pour devenir MSD. 


\section{RÉFÉRENCES}

8. Shi J, Zhao $Y$, Wang $K$, et al. Cleavage of GSDMD by inflammatory caspases determines pyroptotic cell death. Nature 2015 ; 526 : 660-5.

9. Doitsh G, Galloway NK, Geng X, et al. Cell death by pyroptosis drives CD4 T-cell depletion in HIV-1 infection. Nature 2014 ; 505 : 509-14.
10. Mennechet FJ, Tran $\Pi$, Eichholz K, et al. Ebola virus vaccine: benefit and risks of adenovirus-based vectors. Expert Rev Vaccines 2015 ; 14 : 1-8.

11. Gray GE, Moodie Z, Metch B, et al. Recombinant adenovirus type $5 \mathrm{HIV} \mathrm{gag/pol/nef} \mathrm{vaccine} \mathrm{in} \mathrm{South}$ Africa: unblinded, long-term follow-up of the phase 2b HVTN 503/Phambili study. Lancet Infect Dis 2014 ; $14: 388-96$.

\section{NOUVELLE}

\section{Fécondation}

\section{Le noyau spermatique déverrouillé par une thiorédoxine ultraspécialisée}

Béatrice Horard, Benjamin Loppin
12. Duerr A, Huang $Y$, Buchbinder $S$, et al. Extended follow-up confirms early vaccine-enhanced risk of HIV acquisition and demonstrates waning effect over time among participants in a randomized trial of recombinant adenovirus HIV vaccine (Step Study). J Infect Dis 2012 ; $206: 258-66$.
Laboratoire de biométrie et de biologie évolutive, CNRS UMR5558, université Claude Bernard Lyon 1, Villeurbanne, France. benjamin.loppin@univ-lyonl.fr

et permettent son organisation sous une forme extrêmement compacte, qui diffère radicalement de l'organisation en nucléosomes [1].

Si la compaction du noyau spermatique par les protamines contribue certainement aux performances hydrodynamiques du gamète et joue vraisemblablement un rôle protecteur pour son ADN, cette organisation chromatinienne unique n'est pas compatible en l'état avec l'intégration du génome paternel dans le zygote lors de la fécondation. La formation d'un pro-noyau mâle, capable notamment de répliquer son $A D N$, implique en effet le remplacement des protéines SNBP par des histones fournies par le cytoplasme de l'œuf. Notre équipe a d'ailleurs montré, en 2005, que cette étape d'assemblage de novo de la chromatine paternelle mobi-

Figure 1. Distribution des résidus cystéines dans la séquence de trois protéines de la chromatine. L'histone H3.3 est l'une des rares histones à posséder au moins un résidu cystéine (surligné en bleu). Les petites protéines chromosomiques très basiques (SNBP) de mammifères euthériens (protamine $\mathrm{Pl}$ ) et de drosophile (protamin-like $A$ ) sont enrichies en cystéines. Les résidus basiques ( $R, K)$ sont indiqués en gris. 